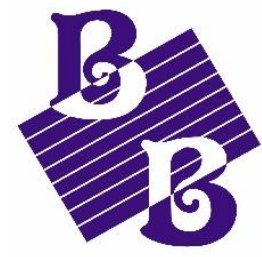

BioBacta
Journal of Bioscience and Applied Research www.jbaar.org

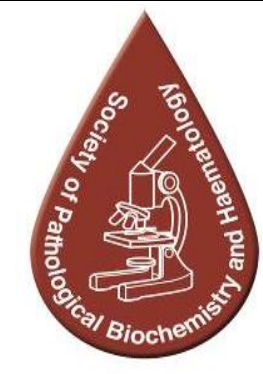

\title{
Evaluation of Blue-Carba Test and ISOPLEX CRE-ART-LAMP assay for rapid screening and characterization of Carbapenemases producing Enterobacteriaceae. Sudan
}

\author{
Omnia M. Hamid ${ }^{1 *}$, Magdi A. Bayoumi² \\ ${ }^{1}$ Department of Medical Microbiology, Faculty of Medical Laboratory Sciences, University of Medical Sciences and \\ Technology (UMST), Khartoum, Sudan \\ ${ }^{2}$ Faculty of Postgraduate Studies, Scientific Research, and Publishing, Ibn Sina University (ISU), Khartoum, Sudan \\ *Corresponding author address \\ Department of Medical Microbiology \\ University of Medical Sciences and Technology (UMST) \\ P.O Box 12810 Khartoum, Sudan \\ Email: omnia.m.hamid@gmail.com \\ ORCID ID: https://orcid.org/0000-0002-1130-0338 \\ DOI: 10.21608/jbaar.2021.174325
}

\section{Abstract}

Objective: We evaluate the performance of the Blue-Carba Test (BCT) and Mast ISOPLEX ®CRE-ART-LAMP assay as quick and affordable assays for the detection of carbapenemase-producing Enterobacteriaceae clinical isolates, Khartoum State-Sudan. Methods: A total of 384 Enterobacteriaceae (122 CPE and 262 non-CPE) clinical isolates were tested for a rapid biochemical method for detection of carbapenemase activity using Blue-Carba Test (BCT), and the Loop-mediated isothermal amplification (LAMP) for carbapenemase target genes detection by MAST ISOPLEX®CRE-ART (19 CPE and 1 non-CPE). Results: The overall sensitivity, specificity and accuracy for carbapenemase detection were 100\% (95\% CI, 97.0\%-100\%), 93.9\% (95\% CI, 90.3\%-96.5\%) and 95.8\% (95\% CI, 93.3\%-97.6\%) for BCT with 80.3\% present positive blue color was appeared after 15 min of test. Interestingly, the Mast ISOPLEX CRE-ART showed specificity 100\%, accuracy 96.7\% (95\% CI, 90.6\%-99.9\%), and sensitivity $94.7 \%$ (95\% CI, $90.3-98.7 \%$ ) for LAMP assay for detection of the target genes and it provides rapid results with a 45-minute. Considering PCR as a standard test for CPE genes detection, the kappa value of BCT and LAMP assays were calculated to be 0.907 and 0.900 respectively, suggesting good agreement between the PCR assay and both selected tests. Conclusion: The promptness and simplicity of both assays make them suitable for inexperienced operators to readily identify carbapenemase production and characterization of major carbapenemase blagenes. Besides exhibits an almost good agreement (Kappa>0.600) emphasizing their high sensitivities and specificities with a rapid and reliable epidemiological screening of carbapenemase production in resource-limited settings.

Keywords: Carbapenemase-producing Enterobacteriaceae, Blue-Carba Test, Mast ISOPLEX CRE-ART-LAMP assay. 


\section{Introduction}

During the last decade, dissemination of carbapenem resistance-carbapenemase-producing organisms including Enterobacteriaceae, Pseudomonas spp. and Acinetobacter spp infections has become one of the most significant threats to public health globally (1). In Sudan, increasing carbapenemase-producing Enterobacteriaceae (CPE) infections associated with hospitalized or community patients posing great challenges to accurate and rapid detection of CPE is imperative to enable specific antibiotic therapy and rapid implementation of infection prevention and control measures and represents a frontier line to prevent the dissemination of resistance determinants (6, 7). As a consequence, the treatment options for CPE infections are often limited, since these organisms usually co-carry resistant determinants to other classes of antibiotics $(4,5)$. The heterogeneity of carbapenemase classes and types leads to a multiplicity of diverse carbapenem hydrolytic efficiencies and resistance phenotypes (5).

In 2017 the World Health Organization (WHO) published its first list of antibiotic 'priority pathogens' that pose the greatest threat to human health, emphasizing the need for laboratories and hospitals to have access to rapid, easy-to-use technologies (8). Besides, The polymerase chain reaction (PCR) combined with sequencing is used as the gold standard for the detection of carbapenemases, its limitations, are required such as expensive equipment, skilled staff, and long detection times, have motivated various scholars and companies to develop new diagnostic methods for the detection of carbapenemases(7). The phenotypic tests detect only carbapenem hydrolysis activity but not the specific gene involved, and most of the developed techniques are target to certain enzymes, with a high possibility of missing non-target or new enzymes $(5,6)$. Herein we chose rapid, inexpensive, and reliable methods to identify CPE which is affordable in our region. The Blue-Carba Test (BCT) is a type of biochemical test for rapid $(<2$ h) carbapenemase detection. The principle of the test is based on the hydrolysis of imipenem by bacterial colonies which can be detected by changes in $\mathrm{pH}$. $(9,10)$. Mast ISOPLEX CRE-ART is an in vitro diagnostic that uses loopmediated isothermal amplification (LAMP) technology to provide rapid molecular identification and differentiation of the seven most prevalent carbapenemase-producing organisms (Enterobacteriaceae, Pseudomonas spp. and Acinetobacter spp) families. For each carbapenemase family targeted by the assay, multiple family members can be detected, as follows: (OXA-48: 31, OXA-23:39, OXA-24/40: 10, KPC: 45, VIM: 64, NDM: 26, IMP: 16) members detected for each gene. (11). The present study was to evaluate the performance of the BCT biochemical method for detection of carbapenemase activity and the LAMP-ISOPLEX®CRE-ART (Mast diagnostica $\mathrm{GmbH}$, Reinfeld, Germany) for the identification of carbapenemases beta-lactam genes (blagenes). Based on these results, a proposed approach was developed for their accurate use for carbapenemase identification.

\section{Materials and Methods}

Bacterial Isolates. We studied a total of non-duplicate 384 Enterobacteriaceae clinical isolates previously isolated from hospitalized patients across three Khartoum state teaching hospitals, Sudan. between April 2018 and October 2019, from sputum, urine, wound exudate, blood, stool, and other body fluids: At each hospital, Enterobacteriaceae were subjected to antimicrobial susceptibility tests by a common protocol, antimicrobial disc diffusion, and classified as resistant or sensitive based on CLSI guidelines M100 $27^{\text {th }}$ (12) at UMST-research laboratory. The molecular conformation of carbapenemase production was performed by the Poirel et al.(13) protocol for a multiplex polymerase chain reaction (PCR) assay for 
the detection and differentiation of the carbapenemases blagenes. The PCR primers were used to target the following groups of carbapenemases; KPC, GES, NDM, VIM, IMP, GIM-1, SIM-1, and OXA-48(13) at the National University Biomedical Research Institute (NUBRI).

Blue-Carba Test. For rapid biochemical detection of $\mathrm{CP}$ directly from bacterial culture, we applied the $\mathrm{J}$. Pires, Â. Novais, L. Peixe (10) protocol; the isolates were cultivated on Luria-Bertani (LB) agar for 18-24 hours. A loopful (about $5 \mu$ l) of pure bacterial culture was added to both test and negative control solution in a 96 well microtiter plate. Incubation of BCT was 30 minutes to two hours at $37^{\circ} \mathrm{C}$. Reading was performed by visually comparing colors between tests well and control well. Change of color of the test well from yellow versus blue, yellow versus green, and green versus blue. In case of a positive result, incubation was stopped after 30 minutes otherwise continued to two hours before a negative result was interpreted. NonCPs failed to change the color of the indicator and both solutions remained either blue $(8,14,15)$. Blue carba test was done in duplicate on the promising CPs isolates after DNA extraction. For a test solution of $0.04 \%$ bromothymol blue adjusted to $\mathrm{pH}=6,0.1 \mathrm{mmol} / \mathrm{liter}$ $\mathrm{ZnSo} 4$ and $3 \mathrm{mg} / \mathrm{ml}$ of imipenem (immediately added before using test) with a final $\mathrm{pH}$ adjusted to 7 was prepared. Negative control of $0.04 \%$ bromothymol blue adjusted to $\mathrm{pH}=7$ was prepared to check the influence of bacterial components on the $\mathrm{pH}$ of the solution (14, $15)$.

Mast ISOPLEX®CRE-ART assay. Bacterial strains were used for establishing the LAMP; the following two positive control isolates were used (1) Klebsiella Spp. strain coproduce: NDM, KPC, OXA-48 and GES (2) E. coli strain coproduce: VIM and IMP, one strain of E.coli non-carbapenemase producer as negative control and $17 \mathrm{CPE}$ strains were used to evaluate the commercial Mast ISOPLEX ®CRE-ART- kit. The assay comprises an eight-tube strip, whereby each of the seven specific carbapenemases (OXA-48, OXA-23, OXA-24/40, KPC, NDM, VIM, and IMP) targets occupies its tube, and tube number eight include an inhibition control for each sample(11). We followed the manufacturer's protocol; DNA was extracted from the bacterial colony cultured Luria-Bertani (LB) agar for overnight incubation at $37^{\circ} \mathrm{C}$ medium by a simple 'boil and spin' step in the buffer provided (provided in Kits). For each tube LAMP pellets in the eight-tube strip, $10 \mu \mathrm{l}$ of the extracted DNA was added directly to run the assay and to determine if the sample being tested is a carbapenemase-producing organism. The LAMP assay was performed as follows; amplification at thermal cycler $65{ }^{\circ} \mathrm{C}$ for $30 \mathrm{mins}$ (cycle time: $1 \mathrm{~min}$ ), and the amplification was detected via the FAM filter (11). A positive result was indicated by a clear difference in intensity output at a given point in time when compared to a no template control (a positive $\mathrm{Ct}<30$ and a negative no $\mathrm{Ct}$ value) (11).

Statistical Analysis. The results of the molecular characterization of the CPE by PCR served as a standard and were compared with the results of the different assays to calculate the sensitivity, specificity, positive and, negative predictive values, and associated confidence intervals (CI) were calculated for each test using MedCalc, an internet-based statistical software (Available from: https://www.medcalc.org/calc/diagnostic_test.php).

The accuracy is the proportion of true-positive and true-negative results of the evaluated assays and indicates the overall probability that a gene was correctly identified. Whilst, Cross tabulation was analyzed to find Kappa value to calculate the test of an agreement by Cohen's Kappa value $\geq 0.6$ were considered statistically significant.

\section{Results}

Performance of the Tested Assays for Detection of CPE 
We have evaluated a BCT against a total 384 Enterobacteriaceae consisting of $(n=122)$ carbapenemase producers and $(n=262)$ noncarbapenemase producers. The distribution of genera was as follows: $E$. coli $(\mathrm{n}=144), K$. pneumoniae $(\mathrm{n}=66)$, E. aerogenes $(\mathrm{n}=34), C$. freundii $(\mathrm{n}=27)$ E. cloacae $(\mathrm{n}=22)$, . mirabilis $(\mathrm{n}=21), P$. stuartii $(\mathrm{n}=19),(\mathrm{n}=17)$ for each M. morganii and Klebsiella Spp and others (8 K. oxytoca, 5 Salmonella Spp and 4 Providencia Spp). Of the $122(31.8 \%)$ CPE isolates were positive for one or more carbapenem resistance genes: the frequency of single carbapenemase genes harbour were IMP ( $\mathrm{n}=$ 37), NDM ( $n=28)$, OXA-48-like $(n=21)$, GES $(n=6)$, KPC (n=5), GIM-1 (n=3), VIM (n=2) and SIM-1 $(n=1)$. Whilst strain coproducing two or more carbapenemase gens NDM+OXA-48(n=5), $(n=3)$ for each IMP+VIM and OXA-48+GES, NDM+KPC+OXA-48+GES ( $\mathrm{n}=2)$ and $(\mathrm{n}=1)$ for each NDM+OXA-48+GES, $\mathrm{NDM}+\mathrm{GES}+\mathrm{OXA}-48+\mathrm{IMP}, \quad \mathrm{VIM}+\mathrm{OXA}-48+\mathrm{GES}$, GES+GIM-1, IMP+KPC and IMP+OXA-48.

Blue-Carba Test: Total of 122 isolates (31.8\%) were correctly identified as CPE using BCT assay, whilst $246(64.0 \%)$ were confirmed negative isolates and 16 (4.2\%) of false-positive isolates those previously carbapenemases negative (non-CPE). Base on detection time $80.3 \%(n=98)$ of CPE strains develop yellow color (positive) with 15 mins, 9.0\% ( $\mathrm{n}=11)$ after 30 mins, 6.6\% ( $n=8)$ strains of (6 strains harbor GES only, and 2 strains co-producer VIM+OXA-48+GES, and GES+GIM-1) after 1 hour and only $4.1 \%(n=5)$ strains of co-producer (GES and other types of carbapenemase blagenes) turn to yellow after $2 \mathrm{hrs}$ of incubation (Table.1). Overall sensitivity of the Blue CARBA test was $100.0 \%$ (95\%CI; 97.0\%-100.0\%) and specificity was $93.9 \%$ (95\% CI; 90.3\%-96.5\%) (Table
3) with accuracy $95.8 \%(95 \% \mathrm{CI} ; 93.3 \%-97.6 \%)$. Interestingly, when comparing the agreement between the BCT and gold standard multiplex PCR tests considering only positive vs. negative results, the tests exhibit an almost good agreement (Kappa=0.907) emphasizing that CBT can be used to detect $\mathrm{CPE}$ given their high sensitivities and specificities.

Mast ISOPLEX®CRE-ART- LAMP assay. As the evaluation panel, 20 Enterobacteriaceae clinical isolates consisting of $(n=19)$ carbapenemase producers and $(n=1)$ non-carbapenemase producers. A total of 17 Enterobacteriaceae clinical isolates $(89.5 .0 \%)$ were correctly identified as carbapenemases producers. Regarding the false-negative results (compared to the known genotypes), the Mast ISOPLEX®CRE-ARTLAMP assay failed to detect two E.coli isolates each with IMP and KPC, producing. Overall, compared to the known genotypes, the Mast ISOPLEX®CRE-ART kit detected $89.5 \%$ of the genes that they were selected to detect (Table.2, Figures 1 and 2) and confirmed 1 (100.0\%) negative isolate (as control negative) was previously carbapenemases negative. The sensitivity of this assay was $94.7 \%$ and specificity was $100.0 \%$, with an accuracy of $96.7 \%$ (95\% CI; 90.6\%-99.9\%) for detecting different carbapenemase types in different Enterobacteriaceae species are shown (Table 2). However, comparing the agreement between the Mast ISOPLEX®CRE-ART by LAMP assay and gold standard multiplex PCR tests considering only positive vs. negative results, the tests exhibit an almost good agreement (Kappa $=0.900)$. The positive result for single carbapenemase genes production was presented in (Figure 1) and Co-producers was identified by the presence of multiple targets (Figure 2) 
Table 1: Performance of the Blue-Carba Test with PCR assay for detection of CPE blagenes with time to positivity:

\begin{tabular}{|c|c|c|c|}
\hline Results of the Blue CARBA test & Negative & Positive & $\begin{array}{l}\text { Time (min) for carbapenemase } \\
\text { detection }\end{array}$ \\
\hline Non-carbapenemase producers $(n=262)$ & 246 & 16 & - \\
\hline Carbapenemase producers $(n=122)$ & $\mathbf{0}$ & 122 & - \\
\hline $\mathrm{KPC}(\mathrm{n}=5)$ & 0 & 5 & $\mathrm{Up}$ to $15 \mathrm{~min}$ \\
\hline GES $(n=6)$ & 0 & 6 & Up to $60 \mathrm{~min}$ \\
\hline $\mathrm{NDM}(\mathrm{n}=28)$ & 0 & 28 & Up to $15 \mathrm{~min}$ \\
\hline $\operatorname{VIM}(n=2)$ & 0 & 2 & Up to $15 \mathrm{~min}$ \\
\hline $\operatorname{IMP}(n=37)$ & 0 & 37 & Up to $15 \mathrm{~min}$ \\
\hline GIM-1 $(n=3)$ & 0 & 3 & $\mathrm{Up}$ to $15 \mathrm{~min}$ \\
\hline $\operatorname{SIM}-1(n=1)$ & 0 & 1 & Up to $60 \mathrm{~min}$ \\
\hline OXA-48 $(n=21)$ & 0 & 21 & Up to $15 \mathrm{~min}$ \\
\hline NDM-1/OXA-48 (n=5) & 0 & 5 & Upto $30 \mathrm{~min}$ \\
\hline OXA-48/GES $(n=3)$ & 0 & 3 & Up to $120 \mathrm{~min}$ \\
\hline $\operatorname{IMP} / \operatorname{VIM}(\mathrm{n}=3)$ & 0 & 3 & Up to $30 \mathrm{~min}$ \\
\hline NDM-1/KPC/OXA-48/GES $(n=2)$ & 0 & 2 & Up to $120 \mathrm{~min}$ \\
\hline NDM-1/GES/OXA-48/IMP $(\mathrm{n}=1)$ & 0 & 1 & Up to $30 \mathrm{~min}$ \\
\hline NDM-1/OXA-48/GES $(n=1)$ & 0 & 1 & Up to $30 \mathrm{~min}$ \\
\hline VIM/OXA-48/GES $(n=1)$ & 0 & 1 & Up to $60 \mathrm{~min}$ \\
\hline $\operatorname{GES} / \operatorname{GIM}(\mathrm{n}=1)$ & 0 & 1 & $\mathrm{Up}$ to $60 \mathrm{~min}$ \\
\hline $\operatorname{IMP} / \mathrm{KPC}(\mathrm{n}=1)$ & 0 & 1 & Up to $15 \mathrm{~min}$ \\
\hline IMP/OXA-48 $(n=1)$ & 0 & 1 & Up to $15 \mathrm{~min}$ \\
\hline
\end{tabular}

Table 2: Assessment of the ISOPLEX CRE-ART ${ }^{\circledR M A S T}$ kit LAMP assay with PCR assay for detection of CPE blagenes:

\begin{tabular}{|c|c|c|c|}
\hline Isolates ID & blagenes types & $\begin{array}{l}\text { CRE-ART kit- } \\
\text { LAMP Result }\end{array}$ & Agreement \\
\hline L18-OTH-3- E. aerogenes & NDM & NDM & Agree \\
\hline L18-IMTH-88-K.pneumoniae & IMP/KPC & KPC & Agree \\
\hline L18-IMTH-97- E. aerogenes & IMP/VIM & IMP/VIM & Agree \\
\hline L18-IMTH-99- E.coli & IMP & - & Disagree \\
\hline L18-OTH-102- K.pneumoniae & IMP & IMP & Agree \\
\hline L18-IMTH-120- M. morganii & VIM & VIM & Agree \\
\hline L18-IMTH-130- Salmonella. Spp & VIM & VIM & Agree \\
\hline L19-IMTH-154- C. freundii & IMP/VIM & IMP/VIM & Agree \\
\hline L18-KNTH-193- E.coli & CNP-blagenes & Non & Agree \\
\hline L18-KNTH-219- E. cloacae & VIM/OXA-48/GES & VIM/OXA-48 & Agree*missed GES \\
\hline L19-KNTH-221- E.coli & IMP/OXA-48 & IMP/OXA-48 & Agree \\
\hline L19-KNTH-273- K.pneumoniae & OXA- 48 & OXA-48 & Agree \\
\hline $\begin{array}{l}\text { L18-OTH-293- Klebsiella. Spp } \\
\text { (Positive C) }\end{array}$ & NDM/KPC/OXA-48/GES & NDM/KPC/OXA-48 & Agree*missed GES \\
\hline L19-OTH-301- E.coli & KPC & - & Disagree \\
\hline L19-OTH-307- K.pneumoniae & $\mathrm{KPC}$ & $\mathrm{KPC}$ & Agree \\
\hline L19-OTH-308- M. morganii & NDM/OXA-48/GES & NDM/OXA-48 & Agree*missed GES \\
\hline L19-OTH-312- E. cloacae & OXA-48 & OXA-48 & Agree \\
\hline L19-OTH-315-E. aerogenes & $\mathrm{KPC}$ & KPC & Agree \\
\hline $\begin{array}{l}\text { L19-OTH-336- E.coli } \\
\text { (positive C) }\end{array}$ & IMP/VIM & IMP/VIM & Agree \\
\hline L19-OTH-381- K.pneumoniae & $\mathrm{KPC}$ & $\mathrm{KPC}$ & Agree \\
\hline L19-KNTH-385- K.pneumoniae & NDM & NDM & Agree \\
\hline Total of LAMP agreement & & & $90.0 \%$ \\
\hline
\end{tabular}

*missed GES; as blaGES was not included with the kits. 
Table 3: Evaluation of the BCT and Mast ISOPLEX®CRE-ART- LAMP assay with PCR assay for detection of CPE:

\begin{tabular}{|c|c|c|c|c|c|c|}
\hline Method & $\begin{array}{l}\text { Sensitivity \% } \\
(95 \% \text { CI })\end{array}$ & $\begin{array}{l}\text { Specificity \% } \\
(95 \% \text { CI })\end{array}$ & $\begin{array}{l}\text { PPV \% } \\
(95 \% \mathrm{CI})\end{array}$ & $\begin{array}{l}\text { NPV \% } \\
(95 \% \mathrm{CI}) \\
\end{array}$ & $\begin{array}{l}\text { Accuracy \% } \\
(95 \% \text { CI })\end{array}$ & $\begin{array}{l}\text { kappa value } \\
(95 \% \mathrm{CI})\end{array}$ \\
\hline Blue CARBA test & $\begin{array}{c}100.0 \% \\
(97.0 \%-100.0 \%)\end{array}$ & $\begin{array}{c}93.9 \% \\
(90.3 \%-96.5 \%)\end{array}$ & $\begin{array}{c}88.4 \% \\
(82.6 \%-92.5 \%)\end{array}$ & $100.0 \%$ & $\begin{array}{c}95.8 \% \\
(93.3 \%-97.6 \%)\end{array}$ & $\begin{array}{c}0.907 \\
(0.86-0.95)\end{array}$ \\
\hline $\begin{array}{l}\text { LAMP ISOPLEX CRE- } \\
\text { ART kit test }\end{array}$ & $\begin{array}{c}94.7 \% \\
(90.3-98.7 \%)\end{array}$ & $100.0 \%$ & $100.0 \%$ & $\begin{array}{c}95.3 \% \\
(92.6 \%-98.5 \%)\end{array}$ & $\begin{array}{c}96.7 \% \\
(90.6 \%-99.9 \%)\end{array}$ & $\begin{array}{c}0.900 \\
(0.87-0.94)\end{array}$ \\
\hline
\end{tabular}

(*) These values are dependent on disease prevalence; PPV: positive productive value; NPV: negative productive value; Cohen's Kappa value $\geq 0.6$ is significant.

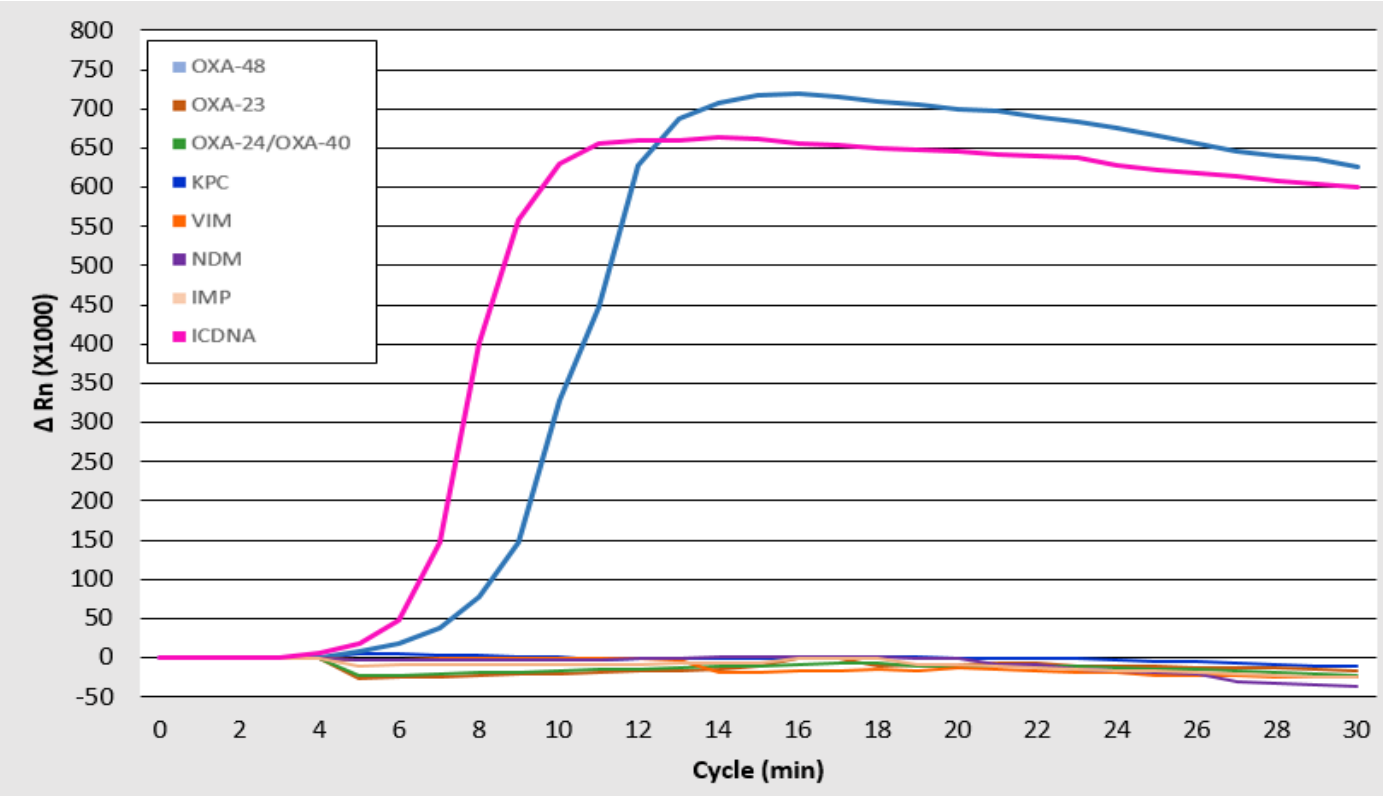

Figure 1: Shows a positive amplification profile for L19-OTH-381- K.pneumoniae producing KPC and the inhibition control DNA (ICDNA) using commercial MAST ISOPLEX CRE-ART kit- LAMP assay. 


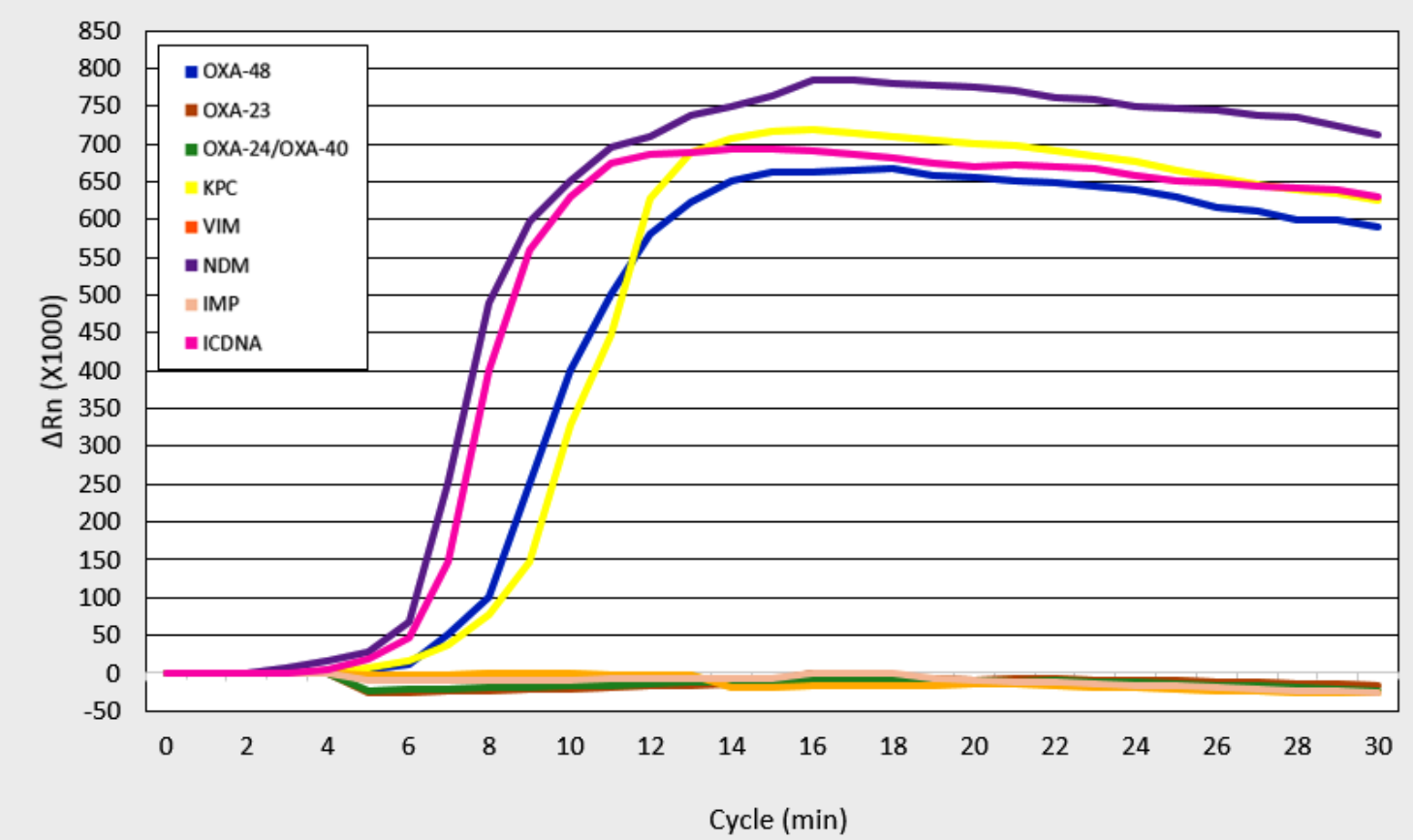

Figure 2: Shows a positive amplification profile for L18-OTH-293- Klebsiella. Spp co-producing OXA-48, NDM, and KPC and the inhibition control DNA (ICDNA), using commercial MAST ISOPLEX CRE-ART kit -LAMP assay.

\section{Discussion}

The accurate and rapid detection of CPE is critical due to the associated therapeutic problems and infection control measures as early identification may optimize antibiotic therapy and minimize unnecessary prescription of medicines (16). In which CPE has already been detected in our region (17) and all over the world (2), with a marked endemicity according to enzyme type introduces resistance to all available antibiotics and makes the choice of antibiotic treatment very limited. Accordingly, the present study aimed to evaluate carbapenemase activity by Blue-Carba Test and characteristics of major carbapenemase blagenes by a commercial Mast ISOPLEX®CRE-ART- LAMPbased assay using Sudanese clinical isolates from hospitalized patients during 2018-2019.

We have evaluated a Blue-Carba Test is a rapid biochemical method that detects carbapenemase activity when the enzyme breaks imipenem's $\beta$-lactam ring, leading to a $\mathrm{pH}$ decrease and consequent color shift of the $\mathrm{pH}$-indicator in solution (10). We found that this test was highly sensitive $(100.0 \%)$ and specific (93.9\%). The BCT proved to be fast (detection observed $\leq 2$ hours), highly sensitive, specific with accuracy up to $95.8 \%$, and very cheap. These findings are in agreement with previous reports $(9,10,14)$. The percentages of agreement between the BCT and multiplex PCR for carbapenemase genes methods for each species were comparatively good, the lowest one recorded for, $86.28 \%$, and the highest for $95.15 \%$ as mentioned before emphasizing that $\mathrm{BCT}$ was able to characterize the $\mathrm{CPE}$ in a simple, rapidly and accessible way, and it can be implemented in clinical laboratories and hospitals with the advantage of having the affordability, accuracy and mainly speed to obtain results when compared traditional methods and molecular techniques for the detection of 
carbapenemase genes are costly, slow and are limited by the targets included(18).

We observed that BCT was delayed to detect class A carbapenemase blaGES, in 13 strains of Enterobacteriaceae producing GES or co-producing GES with one or more carbapenemase genes as the color change to yellow or green to yellow was belated 1-2 hours. A previous study also reported the postponement of color changes or give green to yellow in the detection of some OXA-48 isolates and GES isolates $(9,10)$. Also, we reported 16 out of 262 noncarbapenemase producers as false positive; 7 E.coli, 3 E.aerogenes, 2 for each K.pneumoniae and K.oxytoca, and one for each M.morgenii and P. mirabilis, this might be indicating due to other carbapenems resistance mechanisms rather than the production of carbapenemases(19) like an expression of efflux pumps carbapenems from the bacterial cell or reduction of outer membrane permeability via production of betalactamases $(\mathrm{AmpC})$ in combination with alterations in the bacterial cell membrane (porin mutations in OmpK35 and OmpK36)(19, 20). Any other phenotypic carbapenemase detection methods were not tested in this study this could explain the misclassification.

Commercial Mast ISOPLEX CRE-ART uses LAMP technology to provide rapid detection and characterization of the 'big five' carbapenemase families (KPC, NDM, OXA-48, VIM, IMP) in Enterobacteriaceae and Pseudomonas spp and OXA-23 and OXA-24/OXA-40 in Acinetobacter spp. with a 15minute hands-on time and a 30-minute run time and convenient way. The performance characteristics of the five carbapenemase genes were similar to those detected in the multiplex PCR assay. More specifically, the assays demonstrated diagnostic sensitivity $94.7 \%$ and specificity $100.0 \%$ for all genes and identified a carbapenemase with accuracy $96.7 \%$ of the isolates, except two strains of E.coli produces IMP and KPC were not detected in commercial Mast ISOPLEX CREART- LAMP assay and reported as false-negative results. Moreover, when comparing the agreement with gold slandered assay for carbapenemase genes characterization considering only positive vs. negative results, the tests exhibit an almost perfect agreement [Kappa $=0.900$ (CI 95\% 0.87-0.94)], emphasizing that both can be used to detect and characterize the CPE blagenes given their high sensitivities and specificities. The performance characteristics of the KPC, NDM, VIM, IMP, and OXA-48 commercial Mast ISOPLEX CRE-ART by LAMP assay described in this study support their application for diagnostic and surveillance purposes across resource settings as easyto-use technology.

Limitations regarding Mast ISOPLEX CRE-ART kits; in our study that we studied only 20 Enterobacteriaceae isolates (19 CPE and 1 non-CPE) regarding kits affordability at 2019-2020 due to COVID-19 lockdown. By design, Mast ISOPLEX CRE-ART kits detect only what has included just five major carbapenemase families as in each platform. As such, manufacturers may continuously be updating these platforms mainly with new distributed carbapenemase blagenes such as GES, SIM, and GIM.

Conclusion: Overall, this study confirmed the high sensitivity, and specificity of all the evaluated assays for the detection of CPE and can offer a quicker and more accurate diagnosis than the traditional method. Both tests offer laboratories the opportunity to convey CPE detection by Blue-Carba Test and characterization by Mast ISOPLEX CRE-ART- LAMP assay of five major carbapenemase families in-house in a costeffective manner. We highly recommend applying those assays in clinical practice to reduce the delay in CPE detection and characterization of blagenes, thus providing physicians and infection control practitioners with better knowledge of the local epidemiology and to proper use of antimicrobial agents, besides improved patient outcome in our sitting regarding resources was limited. 


\section{Conflicts of Interest.}

The authors declare no conflict of interest.

\section{Acknowledgements.}

We would like to acknowledge Dr. Sieghard Frischmann for his offering the ISOPLEX CRE-ART. Kits (Mast Diagnostica $\mathrm{GmbH}$ ). In addition $\mathrm{Mr}$. Osama Mohammed for molecular techniques workflow at the National University Biomedical Research Institute (NUBRI Khartoum-Sudan).

\section{Funding.}

Not funded (corresponding author fund all work as part of $\mathrm{PhD}$ degree)

Data availability.

All data generated or analyzed during this study are included in this published article (are available from the corresponding author on reasonable request)

\section{Author information.}

$\mathrm{OMH}$, Conceived, designed, and performed the experiments. $\mathrm{OMH}$ writing of the manuscript and MAHB manuscript revised and supervised the research work. All authors read and approved the final manuscript.

\section{Corresponding author.}

Correspondence to Omnia.M.Hamid

\section{References}

1. Santajit S, Indrawattana N. Mechanisms of antimicrobial resistance in ESKAPE pathogens. BioMed research international. 2016;2016.

2. Tängdén T, Giske C. Global dissemination of extensively drug-resistant carbapenemase-producing $\mathrm{E}$ Enterobacteriaceae: clinical perspectives on detection, treatment and infection control. Journal of Internal Medicine. 2015;277(5):501-12.

3. Albiger B, Glasner C, Struelens MJ, Grundmann H, Monnet DL. Carbapenemase-producing
Enterobacteriaceae in Europe: assessment by national experts from 38 countries, May 2015. Eurosurveillance. 2015;20(45):30062.

4. Tzouvelekis L, Markogiannakis A, Piperaki E, Souli M, Daikos G. Treating infections caused by carbapenemase-producing Enterobacteriaceae. Clinical Microbiology and Infection. 2014;20(9):862-72.

5. Hrabák J, Chudáčková E, Papagiannitsis C. Detection of carbapenemases in Enterobacteriaceae: a challenge for diagnostic microbiological laboratories. Clinical Microbiology and Infection. 2014;20(9):83953.

6. Dortet L, Bréchard L, Cuzon G, Poirel L, Nordmann P. Strategy for rapid detection of carbapenemase-producing Enterobacteriaceae. Antimicrobial agents and chemotherapy. 2014;58(4):2441-5.

7. Khalifa HO, Okanda T, Abd El-Hafeez AA, Abd El Latif A, Habib AG, Yano H, et al. Comparative evaluation of five assays for detection of carbapenemases with a proposed scheme for their precise application. The Journal of Molecular Diagnostics. 2020;22(9):1129-38.

8. Organization WH. Guidelines for the prevention and control of carbapenem-resistant Enterobacteriaceae, Acinetobacter baumannii and Pseudomonas aeruginosa in health care facilities. 2017.

9. Pires J, Tinguely R, Thomas B, Luzzaro F, Endimiani A. Comparison of the in-house made CarbaNP and Blue-Carba tests: considerations for better detection of carbapenemase-producing Enterobacteriaceae. Journal of microbiological methods. 2016;122:33-7.

10. Pires J, Novais A, Peixe L. Blue-carba, an easy biochemical test for detection of diverse carbapenemase producers directly from bacterial 
cultures. Journal of clinical microbiology. gram-negative bacilli. Journal of clinical microbiology. 2013;51(12):4281-3. 2015;53(6):1996-8.

11. Group BHitMPSaM. Using molecular LAMP to curtail the global threat of carbapenem resistance 2019 ; Available from: https://www.pathologyinpractice.com/story/31502/usi ng-molecular-lamp-to-curtail-the-global-threat-ofcarbapenem-resistance.

12. Abbey TC, Deak E. What's New from the CLSI Subcommittee on Antimicrobial Susceptibility Testing M100. Clinical Microbiology Newsletter. 2019;41(23):203-9.

13. Poirel L, Walsh TR, Cuvillier V, Nordmann P. Multiplex PCR for detection of acquired carbapenemase genes. Diagnostic microbiology and infectious disease. 2011;70(1):119-23.

14. Nastro M, Ayora M, García S, Vay C Famiglietti Á, Rodriguez CH. Rapid Blue-Carba test: reduction in the detection time of carbapenemases performed from a 4-hour bacterial lawn. Journal of Chemotherapy. 2017;29(3):150-3.

15. Pasteran F, Veliz O, Ceriana P, Lucero C, Rapoport M, Albornoz E, et al. Evaluation of the BlueCarba test for rapid detection of carbapenemases in
16. Chan WW, Campbell L, Doyle D, Pitout JD. Rapid detection of Enterobacterales that produce carbapenemases. Diagnostic microbiology and infectious disease. 2020;98(2):115120.

17. Dahab R, Ibrahim AM, Altayb HN. Phenotypic and genotypic detection of carbapenemase enzymes producing gram-negative bacilli isolated from patients in Khartoum State. F1000Research. 2017;6(1656):1656.

18. Sun K, Xu X, Yan J, Zhang L. Evaluation of six phenotypic methods for the detection of carbapenemases in Gram-negative bacteria with characterized resistance mechanisms. Ann Lab Med. 2017;37(4):305-12.

19. Nordmann P, Poirel L, Dortet L. Rapid detection of carbapenemase-producing Enterobacteriaceae. Emerging infectious diseases. 2012;18(9):1503.

20. Logan LK, Weinstein RA. The epidemiology of carbapenem-resistant Enterobacteriaceae: the impact and evolution of a global menace. The Journal of infectious diseases. 2017;215(suppl_1):S28-S36. 